\title{
Risk factors of para-aortic lymph node metastasis in patients with endometrial carcinoma
}

\author{
Jiong Bo Liao\#, Bing Li", Bing Yi Yang, Yue Shi, Xue Zhen Luo, Chao Wang*, Xiao Jun Chen* \\ Department of Obstetrics and Gynecology, Obstetrics and Gynecology Hospital, Fudan University, Shanghai 200011, China; \\ \# These authors contributed equally to this work. * Corresponding Author
}

\begin{abstract}
Objectives: To explore the risk factors of para-aortic lymph node (PALN) metastasis in patients with endometrial carcinoma.

Methods: A retrospective analysis of patients with endometrial carcinoma admitted to Obstetrics and Gynecology Hospital of Fudan University from January 1st, 2009 to April 30th, 2017. 514 cases with comprehensive staging surgery were included. Univariate and multivariate analysis were performed with SPSS 23.0 and $\mathrm{R}$ software in order to explore the relationships between para-aortic lymphatic metastasis and clinical pathological factors. Meanwhile, a meta-analysis was performed to investigate the risk factors of PALN metastasis in endometrial cancer, which were reported in previous literature.
\end{abstract}

\section{Results:}

$79(15.3 \%)$ cases had pelvic lymph nodes (PVLN) metastasis, 59 (11.5\%) PALN metastasis, and 21 of 59 cases were PALN metastasis without PVLN metastasis. According to the "Mayo" risk stratification system, we divided our patients into three groups, the ratio PVLN metastasis and PALN metastasis were $1.9 \%$ and $0.9 \%$ in low-risk group, $14.9 \%$ and $11.4 \%$ in intermediate-risk group, $23.8 \%$ and $18.3 \%$ in high-risk group, respectively

(figure 1). The multivariate logistic regression model showed that, PVLN metastasis was the most relative factor of PALN metastasis with the OR of 7.21. Cervical stromal invasion $(\mathrm{Cl})$ and TSIZE followed it with the OR 4.022 and 2.855 (figure 2). The meta-analysis we did with published references from 1988 to 2017 in the database showed that adnexal involvement $(\mathrm{OR}=6.62$, $95 \% \mathrm{Cl}[4.84-9.06])$, cervical stromal invasion (OR=4.47 95\%Cl[3.33-6.01]), peritoneal cytology positive ( $\mathrm{OR}=3.3495 \% \mathrm{Cl}[2.24-4.97]$ ) , LVSI positive (OR=6.08 $95 \% \mathrm{Cl}[4.17-8.86])$ and PVLN positive (OR=36.11 $95 \% \mathrm{Cl}[25.93-51.11])$ were risk factors of PALN metastasis (figure 3).

Conclusion: adnexal involvement, deep myometrium invasion, peritoneal cytology positive, CI, TSIZE, LVSI positive and PVLN positive increased the risk of PALN metastatic. Our data indicated $\mathrm{Cl}$, TSIZE, LVSI positive, PVLN positive were the top 4 risk factors of PALN metastatic, especially PVLN positive. We commend PALN dissection should be performed for those who have the high-risk factors mentioned above.

\begin{tabular}{ccccccccc}
\hline Risk factors & df & Beta & S.E. & Wald & P & OR & \multicolumn{2}{c}{$95 \%$ Cl for Wald } \\
& & & & & & & lower & upper \\
\hline Intercept & 1 & -8.3914 & 0.8153 & 105.932 & $<.0001$ & & & \\
PVLN & 1 & 1.9764 & 0.3526 & 31.4229 & $<.0001$ & 7.217 & 3.616 & 14.403 \\
CI & 1 & 1.3917 & 0.3903 & 12.7115 & 0.0004 & 4.022 & 1.871 & 8.643 \\
TSIZE & 1 & 1.049 & 0.3872 & 7.3382 & 0.0068 & 2.855 & 1.336 & 6.098 \\
\hline
\end{tabular}

Figure 2. Multivariate analysis of risk factors associated with PALN metastasis with multivariate logistic regression model.

This study was supported by National Natural Science Foundation of China (\#8 I772777), Shanghai Pujiang Program (I7PJ I40 I400) and Shanghai Science and technology committee medical guidance program (\#|8411963700)

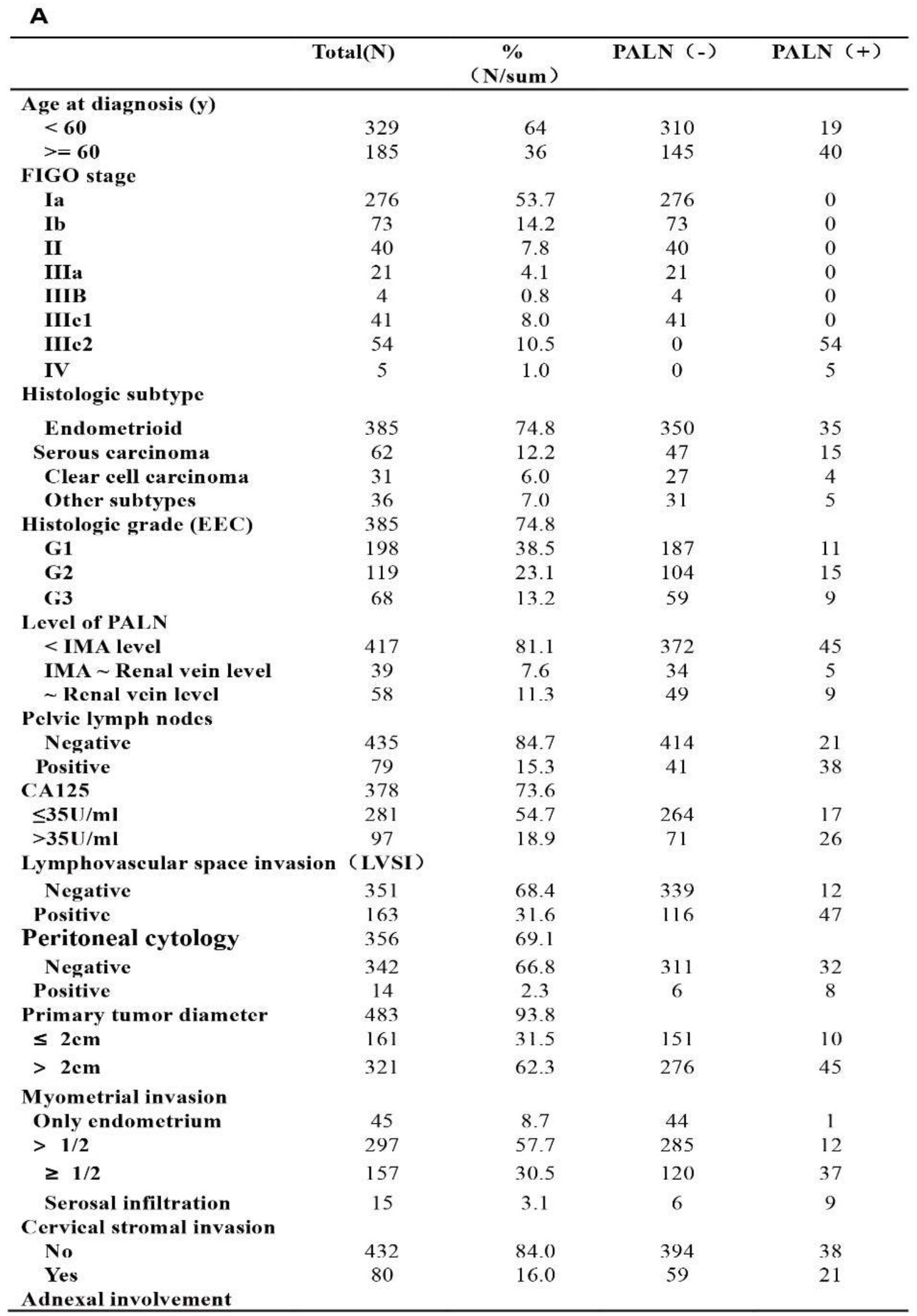

B

\begin{tabular}{cccc}
\hline B & \multicolumn{3}{l}{} \\
Risk group & Sum & $\begin{array}{c}\text { Metastatic PVLN } \\
\%(\mathrm{~N})\end{array}$ & $\begin{array}{c}\text { Metastatic PALN } \\
\%(\mathrm{~N})\end{array}$ \\
\hline Low & 105 & $1.9 \%(2)$ & $0.9 \%(1)$ \\
Intermediate & 120 & $6.7 \%(8)$ & $4.2 \%(5)$ \\
High & 289 & $23.8 \%(69)$ & $18.3 \%(53)$ \\
& $\mathrm{P}<0.001$ & $\mathrm{P}<0.001$ & $\mathrm{P}<0.001$ \\
\hline
\end{tabular}

Figure 1. Clinical pathological characteristic of patients and lymph node metastasis. (A) Clinical pathological characteristic of patients rate in different groups. (B) The lymph node metastasis rate in different groups. Legend: $P A L N=$ para-aortic lymph nodes, $P V L N=$ pelvic lymph nodes, IMA= inferior mesenteric artery, MELF in Itration= microcystic, elongated and fragmented pattern in Itration. Low risk group: a, Endometrioid, grade 1-2, $<1 / 2$ myometrial invasion, Primary tumor diameter $\leq 2 \mathrm{~cm}$; $b$, Endometrioid, Tumor limited in only endometrium (grade 1-3, Primary tumor diameter no limit) ; Intermediate risk group: Endometrioid, grade $1-2,<1 / 2$ myometrial invasion, Primary tumor diameter $>2 \mathrm{~cm}$; High risk group: a, Non-endometrioid (serous or clear-cell or undifferentiated carcinoma, or carcinosarcoma; b, Endometrioid G3 with myometrial invasion; $\geq 1 / 2$ myometrial invasion.
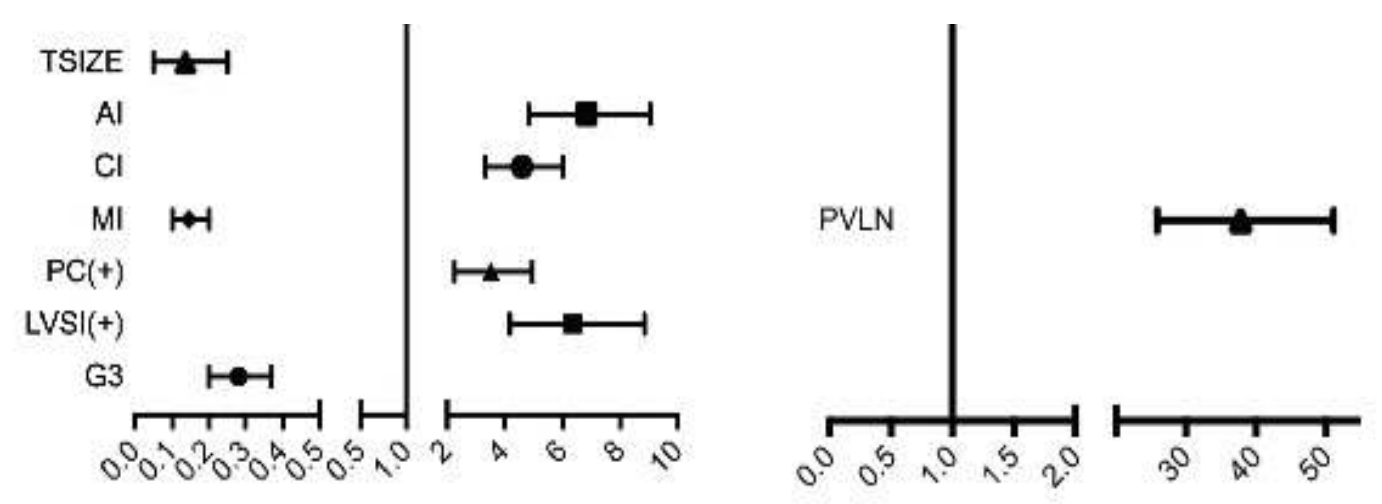

Figure 3. Meta-analysis of risk factors for PALN metastasis in endometrial cancer patients with comprehensive surgical staging. A meta-analysis of risk factors for PALN metastasis was conducted, which included 8 studies. 